\title{
Twenty-Four-Hour Urinary Aldosterone Predicts Inappropriate Left Ventricular Mass Index in Patients with Primary Aldosteronism
}

\author{
Chi-Sheng Hung, ${ }^{1,2}$ Yi-Lwun Ho, ${ }^{1,2}$ Yi-Yao Chang, ${ }^{2}$ Vin-Cent $W u,{ }^{2}$ Xue-Ming Wu, ${ }^{3}$ \\ Jen-Kuang Lee, ${ }^{4}$ Shih-Chieh Chueh, ${ }^{5,6}$ Yen-Hung Lin, ${ }^{2}$ Yuan-Shian Changh, \\ Shao-Yu Yang, ${ }^{2}$ Ya-Hui Hu, ${ }^{8}$ Ming-Jai Sui, ${ }^{9}$ Ming-Fong Chen, ${ }^{2}$ and Kwan-Dun Wu ${ }^{2}$ \\ ${ }^{1}$ Telehealth Center, National Taiwan University Hospital, No. 7 Chung-Shan S. Road, Taipei 100, Taiwan \\ ${ }^{2}$ Department of Internal Medicine, National Taiwan University Hospital and National Taiwan University College of Medicine, \\ No. 7 Chung-Shan S. Road, Taipei 100, Taiwan \\ ${ }^{3}$ Department of Internal Medicine, Taoyuan General Hospital, Taoyuan 330, Taiwan \\ ${ }^{4}$ Department of Laboratory Medicine, Far Eastern Memorial Hospital, New Taipei City 220, Taiwan \\ ${ }^{5}$ Department of Urology, National Taiwan University Hospital and National Taiwan University College of Medicine, Taipei 100, Taiwan \\ ${ }^{6}$ Department of Urology, Cleveland Clinic, Cleveland, OH 44011, USA \\ ${ }^{7}$ Department of Internal Medicine, Postal Hospital, Taipei 100, Taiwan \\ ${ }^{8}$ Internal Medicine, Buddhist Tzu Chi General Hospital, New Taipei City 231, Taiwan \\ ${ }^{9}$ Institute of Pharmacology, National Taiwan University Medical College, Taipei 100, Taiwan
}

Correspondence should be addressed to Yen-Hung Lin; austinr3@yahoo.com.tw

Received 4 October 2013; Accepted 18 November 2013

Academic Editors: D. C. Gaze and N. Mansencal

Copyright (C) 2013 Chi-Sheng Hung et al. This is an open access article distributed under the Creative Commons Attribution License, which permits unrestricted use, distribution, and reproduction in any medium, provided the original work is properly cited.

Objective. Primary aldosteronism (PA) is associated with inappropriate left ventricular hypertrophy (LVH) in relation to a given gender and body size. There is no ideal parameter to predict the presence of LVH or inappropriate LVH in patients with PA. We investigate the performance of 24-hour urinary aldosterone level, plasma renin activity and aldosterone-to-renin ratio on this task. Methods. We performed echocardiography in 106 patients with PA and 31 subjects with essential hypertension (EH) in a tertiary teaching hospital. Plasma renin activity, aldosterone concentration, and 24-hour urinary aldosterone level were measured. Results. Only 24-hour urinary aldosterone was correlated with left ventricular mass index (LVMI) and excess LVMI among these parameters. The multivariate analysis revealed the urinary aldosterone level as an independent predictor for LVMI and excess LVMI. Analyzing the ability of urinary aldosterone, plasma aldosterone concentration, and plasma aldosterone-to-renin ratio to identify the presence of LVH (ROC AUC $=0.701,0.568,0.656$, resp.) and the presence of inappropriate LV mass index (defined as measured LVMI in predicting LVMI ratio $>135 \%$ ) (ROC area under curve $=0.61,0.43,0.493$, resp.) revealed the better performance of 24 hour urinary aldosterone. Conclusions. In conclusion, 24-hour urinary aldosterone level performed better to predict the presence of LVH and inappropriate LVMI in patients with PA.

\section{Introduction}

Primary aldosteronism (PA), characterized by an inappropriate production of aldosterone, can be found in $5-13 \%$ of patients with hypertension $[1,2]$. PA was once thought to be a rare condition. However, new evidence suggests that
PA is the most frequent cause of secondary hypertension [3]. Patients with PA have an inappropriate degree of left ventricular concentric remodeling compared with patients with essential hypertension of a similar level of blood pressure [4-6]. Aldosterone not only stimulates cardiomyocyte growth and hypertrophy but also increases the production 
of extracellular matrix; thus, leads to increased myocardial fibrosis [7]. Left ventricular hypertrophy (LVH) is a compensatory response to the increased afterload. Initially, $\mathrm{LVH}$ reduce the increased stress on the myocardium. However, the left ventricular mass (LVM) may increase disproportionately to the hemodynamic load in patients with PA [4]. The increase in aldosterone levels has been demonstrated to be a possible contribution to this inappropriate increase in LV mass [4]. The appropriateness of LVM to loading condition and body size can be estimated by gender, body height, blood pressure, and stroke volume $[4,8]$. In patients with essential hypertension, the presence of inappropriate LVM for a given workload is associated with a higher prevalence of low LV systolic contractility and abnormal relaxation, which suggests a higher risk of transition from compensatory LVH into symptomatic heart failure [8]. The long-term effect of inappropriate LVM in patients with PA is still unknown.

Although aldosterone is directly involved in the pathogenesis of LVH in patients with PA, the plasma aldosterone concentration (PAC), plasma renin activity (PRA), and aldosterone-to-renin ratio were only weakly correlated with LVM in these patient groups [4,9]. This may be partly caused by the circadian fluctuation of plasma level of aldosterone, with a peak in the early morning. The measurement of 24-hour urinary aldosterone has a potential advantage to estimate the integrated daily exposure to aldosterone, and to avoid the large fluctuation in the circulating plasma aldosterone level. In patients with essential hypertension, 24hour urine aldosterone level has a better correlation with LVM than plasma aldosterone level and in LV mass [10, 11]. The 24-hour urinary aldosterone has been shown to have a weak correlation with the degree of LVM inappropriateness $(r=0.19 ; P=0.05)$ in patients with PA [4]. However, the comparison of the performance between plasma aldosterone and 24-hour urinary aldosterone was not conducted in this study. There is still no ideal parameter to predict the presence of LVH or inappropriate LVH in patients with PA. We design this study to elucidate which parameter is more associated with impaired LV geometry in patients with PA and can predict the presence of LVH and inappropriate LVMI more accurately.

\section{Methods}

2.1. Patients. This cross-sectional study enrolled 106 patients diagnosed with PA from October 2006 to March 2010. The patients were evaluated and registered in the Taiwan Primary Aldosteronism Investigation (TAIPAI) database. The database was constructed for quality assurance in one medical center (National Taiwan University Hospital, Taipei, Taiwan) and its three branch hospitals in different cities (National Taiwan University Hospital Yun-Lin branch, YunLin, southern Taiwan; Far-Eastern Memorial Hospital, Taipei; and Taoyuan General Hospital, Taoyuan, mid Taiwan) [12, 13]. The patients were divided into two groups by median level of 24-hour urinary aldosterone (group 2 and group3, with lower and higher urinary aldosterone level, resp.). Another 31 patients with essential hypertension (group 1) were enrolled from the outpatient department of our hospital for comparison. The medical history of the subjects, including demography and current medication, was recorded. The diagnosis of essential hypertension was made by exclusion according to standard algorithms, based on clinical history and biochemical investigations of all detectable forms of secondary hypertension.

The serum biochemistry was measured when these patients were first evaluated at National Taiwan University Hospital. The concentration of aldosterone was measured by radioimmunoassay with commercial kits (Aldosterone Maia Kit, Adaltis Italia S.P.A., Bologna, Italy) [14]. The limit of detection was $10.0 \mathrm{pg} / \mathrm{mL}$ at a $90 \%$ confidence interval. The normal range of aldosterone is $70-350 \mathrm{pg} / \mathrm{mL}$ in the upright position. The plasma renin activity was measured by the generation of angiotensin I in vitro using a commercially available RIA kit (Stillwater, MN, USA). Its normal range was $2.63 \pm 1.32 \mathrm{ng} / \mathrm{mL} / \mathrm{h}$ in the upright position. The intra-assay coefficient of variability for the PRA assay was $1.9(5.0 \%)$ (mean (SD)); interassay coefficient of variability $4.5(5.2 \%)$. The echocardiography and the measurement of serum biochemistry were performed at the first evaluation (within one month period from the outpatient clinic visit). Urine samples were collected and stored at $-20 \mathrm{C}$ until the measurements were performed. It was acid hydrolysis and then followed the assay procedure as serum. Daily urine amount was recorded and $24 \mathrm{hr}$ urinary aldosterone level was calculated by multiplying urine aldosterone value by the daily urine amount. When interpreting the results of a 24hour urine collection, we assess the adequacy of collection by quantifying the 24-hour urine creatinine excretion. The $24-$ hour urine creatinine excretion was between 15 and $20 \mathrm{mg} / \mathrm{kg}$ body weight.

2.2. Diagnostic Criteria for PA. The diagnosis of PA was established in patients with hypertension if all of the following criteria were met: (a) evidence of autonomous aldosterone production based on a post captopril ARR of greater than $35 \mathrm{ng} / \mathrm{dL}$ per $\mathrm{ng} / \mathrm{mL} / \mathrm{h}$ and a plasma aldosterone concentration $>10 \mathrm{ng} / \mathrm{dL}$; (b) a positive salt infusing test or TAIPAI score $>0.960$ (60\% probability) [14]; (c) evidence of an adenoma, or diffuse enlargement of the adrenal glands on computerized tomography; and (d) a positive dexamethasone suppression test or lateralization of aldosterone secretion on adrenal vein sampling.

Hypertensive patients with screening ARR of $<30 \mathrm{ng} / \mathrm{dL}$ per $\mathrm{ng} / \mathrm{mL} / \mathrm{h}$, plasma aldosterone concentration $<25 \mathrm{ng} / \mathrm{dL}$, and a negative salt loading test result were diagnosed with essential hypertension.

2.3. Echocardiography. A Hewlett-Packard 5500 ultrasound system with a S3 transducer $(1.0-3.0 \mathrm{MHz})$ was used in this study. Transthoracic echocardiographic images were acquired in the fundamental imaging mode. Two-dimensional, M-mode Doppler and tissue Doppler ultrasonography were performed in each patient. Chamber dimension, wall thickness, and left ventricular ejection fraction (M-mode) were measured according to the guidelines of the American Society of Echocardiography by one experienced cardiologist [15]. Another cardiologist measured the echocardiographic 
TABLE 1: Clinical data of patients.

\begin{tabular}{|c|c|c|c|c|}
\hline Group & $1(n=31)$ & $2(n=53)$ & $3(n=53)$ & $P$ \\
\hline Age, years, mean (SD) & $50.5(15.6)$ & $50.9(11.4)$ & $46.9(12.6)^{\S}$ & 0.209 \\
\hline Men, $n(\%)$ & $11(35.5)$ & $26(47.3)$ & $24(43.6)$ & 0.569 \\
\hline History of hypertension, years, mean (SD) & $5.2(5.0)$ & $8.0(7.6)$ & $6.6(7.8)$ & 0.251 \\
\hline Body weight, kg, mean (SD) & $62.7(12.0)$ & $67.2(14.0)$ & $66.9(13.0)$ & 0.293 \\
\hline Body height, cm, mean (SD) & $162.2(9.7)$ & $161.0(8.3)$ & $161(8.8)$ & 0.744 \\
\hline Body mass index, $\mathrm{kg} / \mathrm{m}^{2}$, mean (SD) & $23.7(2.6)$ & $25.9(4.0)^{\dagger}$ & $25.7(3.5)^{9}$ & 0.019 \\
\hline Blood urea nitrogen, mg/dL, mean (SD) & $13.6(3.1)$ & $14.0(4.2)$ & $12.1(3.8)^{\S}$ & 0.052 \\
\hline Creatinine, $\mathrm{mg} / \mathrm{dL}$, mean (SD) & $0.94(0.17)$ & $0.97(0.23)$ & $0.91(0.24)$ & 0.472 \\
\hline SBP, mmHg, mean (SD) & $146(17)$ & $152(22)$ & $153(22)$ & 0.293 \\
\hline DBP, mmHg, mean (SD) & $87(11)$ & $94(13)^{\dagger}$ & $93(14)$ & 0.056 \\
\hline Serum potassium, mEq/L, mean (SD) & $4.21(0.33)$ & $3.53(0.67)^{\dagger}$ & $3.47(0.78)^{9}$ & $<0.001$ \\
\hline $\mathrm{PAC}, \mathrm{ng} / \mathrm{dL}$, mean (SD) & $24.3(11.1)$ & $48.9(39.5)^{\dagger}$ & $57.0(37.6)^{9}$ & $<0.001$ \\
\hline PRA, ng/mL/h, mean (SD) & $7.5(8.8)$ & $0.7(0.9)^{\dagger}$ & $0.8(1.6)$ & $<0.001$ \\
\hline $\mathrm{PAC}^{*}$, mean $(\mathrm{SD})$ & $1.34(0.18)$ & $1.59(0.26)^{\dagger}$ & $1.67(0.27)^{9}$ & $<0.001$ \\
\hline $\mathrm{PRA}^{*}$, mean $(\mathrm{SD})$ & $0.53(0.6)$ & $-0.52(0.7)^{\dagger}$ & $-0.63(0.8)^{9}$ & $<0.001$ \\
\hline 24-hour urinary aldosterone level, mean (SD) & $6.5(2.4)$ & $6.1(1.4)$ & $23.7(12.4)^{9 \$}$ & $<0.001$ \\
\hline 24-hour urinary aldosterone level ${ }^{*}$, mean (SD) & $0.79(0.14)$ & $0.77(0.11)$ & $1.31(0.22)^{9 \$}$ & $<0.001$ \\
\hline Number of hypertension medication, mean (SD) & $1.7(0.9)$ & $2.0(1.0)$ & $1.8(1.0)$ & 0.8 \\
\hline \multicolumn{5}{|l|}{ Hypertension medication: } \\
\hline $\mathrm{CCB}, n(\%)$ & $17(55)$ & $30(57)$ & $29(55)$ & 0.598 \\
\hline ACEI or ARB, $n(\%)$ & $19(62)$ & $13(25)^{\dagger}$ & $15(28)^{9}$ & 0.004 \\
\hline Thiazide, $n(\%)$ & $3(10)$ & $2(3.7)$ & $5(9.4)$ & 0.547 \\
\hline$\alpha$-blocker, $n(\%)$ & $3(10)$ & $14(26.4)^{\dagger}$ & $22(41.5)^{9}$ & 0.006 \\
\hline$\beta$-blocker, $n(\%)$ & $9(29)$ & $28(52.8)^{\dagger}$ & $19(35.8)^{\S}$ & 0.01 \\
\hline Spironolactone, $n(\%)$ & $0(0)$ & $23(43)^{\dagger}$ & $25(47.2)^{9}$ & $<0.001$ \\
\hline Adenoma, $n(\%)$ & & $30(58)$ & $46(87)^{\S}$ & $<0.001$ \\
\hline
\end{tabular}

${ }^{*} \log$-transformed.

** SBP: systolic blood pressure; DBP: diastolic blood pressure; PAC: plasma aldosterone concentration; PRA: plasma renin activity; CCB: calcium channel blocker; ACEI: Angiotensin converting enzyme inhibitor; ARB: angiotensin receptor blocker.

${ }^{\dagger}$ Group 2 versus group $1, P<0.05$.

'Group 3 versus group 1, $P<0.05$.

${ }^{\S}$ Group 3 versus group 2, $P<0.05$.

data off-line to confirm the reproducibility. The interobserver and intraobserver variability were $8.0 \%$ and $8.7 \%$, respectively.

Measured LVMI was derived with echocardiography according to the formula of Devereux and Reichek LV mass = $1.04 \times[$ (septal thickness + LV end-diastolic diameter + posterior wall thickness $\left.)^{3}-(\text { LV end-diastolic diameter })^{3}\right]-$ 13.6 (gm) [16]. The predicted LVMI was estimated by the equation derived previously: Predicted $\mathrm{LVM}=55.37+6.64 \times$ height $\left(\mathrm{m}^{2.7}\right)+0.64 \times$ stroke work $-18.07 \times$ gender (where gender was coded as male $=1$ and female $=2$ ) [7]. Left ventricle volume was calculated with Tericholz's formula; stroke work was calculated as systolic blood pressure (in $\mathrm{mmHg}) \times$ stroke volume $\times 0.0144$ [4]. The excess LVMI was defined as measured LVMI-predicted LVMI. The presence of inappropriate LVMI was defined as positive if measured LVMI to predicted LVMI ratio was greater than 135\% [4]. LVH was defined according to Devereux's criteria: LVMI $\geqq$ $134 \mathrm{~g} \mathrm{~m}^{-2}$ in men and $110 \mathrm{~g} \mathrm{~m}^{-2}$ in women [17].
2.4. Statistical Analysis. All of the continuous variables were expressed as mean \pm standard deviation (SD). Comparisons for continuous data between the three groups were made by one-way ANOVA. Differences between proportions were assessed by Chi-square test. Pearson's correlation test was used to analyze the correlation between LVMI and continuous and categorical parameters. Data of urine aldosterone concentration, PAC, PRA, and ARR were log-transformed before the correlation study due to the nonnormality which was determined by the Kolmogorov-Smirnov test. Before further analysis, the log-transformed data was tested again to assure the normality of distribution. A stepwise multivariate linear regression analysis was used to evaluate the relationship between LVMI and different clinical or laboratory variables. The performances of 24-hour urinary aldosterone, plasma aldosterone level, and plasma aldosterone-to-renin activity ratio (ARR) to predict the presence of $\mathrm{LVH}$ and inappropriate LVMI were determined by the receiver-operator characteristic (ROC) curve analysis. A two-tailed $P$ value below 0.05 was regarded as significant. The statistical analyses 
TABLE 2: Echocardiographic data.

\begin{tabular}{|c|c|c|c|c|}
\hline Group & 1 & 2 & 3 & $P$ \\
\hline IVST, mean (SD) & $10.3(2.2)$ & $11.4(2.0)^{\dagger}$ & $12.4(2.2)^{95}$ & $<0.001$ \\
\hline LVPWT, mean (SD) & $9.4(1.3)$ & $10.8(1.7)^{\dagger}$ & $11.6(1.7)^{9 \$}$ & $<0.001$ \\
\hline LVEDD, mean (SD) & $45.5(4.8)$ & $45.6(5.2)$ & $46.4(5.2)$ & 0.649 \\
\hline LVESD, mean (SD) & $26.0(5.1)$ & $28.4(4.7)^{\dagger}$ & $27.9(4.7)$ & 0.098 \\
\hline LVM, mean (SD) & $180.1(55.3)$ & $220.5(85.1)$ & $249.7(76.0)^{9}$ & $<0.001$ \\
\hline LVMI, g/m², mean (SD) & $106.3(27.1)$ & $127.0(42.4)^{\dagger}$ & $144.3(38.0)^{95}$ & $<0.001$ \\
\hline LVEF, mean (SD) & $73.0(9.1)$ & $67.1(7.5)^{\dagger}$ & $69.6(7.6)$ & 0.008 \\
\hline E, mean $(\mathrm{SD})$ & $74.6(14.9)$ & $75.0(16.4)$ & $72.4(17.2)$ & 0.695 \\
\hline $\mathrm{A}$, mean $(\mathrm{SD})$ & $70.2(18.1)$ & $74.2(14.0)$ & $73.8(19.6)$ & 0.550 \\
\hline $\mathrm{E} / \mathrm{A}$, mean $(\mathrm{SD})$ & $1.14(0.34)$ & $1.04(0.28)$ & $1.03(0.33)$ & 0.293 \\
\hline $\mathrm{E}^{\prime}$ (septal), mean (SD) & $8.5(1.7)$ & $7.5(2.5)^{\dagger}$ & $7.1(2.1)^{9}$ & 0.018 \\
\hline $\mathrm{A}^{\prime}$ (septal), mean (SD) & $10.5(1.8)$ & $11.0(2.6)$ & $10.5(2.1)$ & 0.494 \\
\hline Stroke volume index $\left(\mathrm{mL} / \mathrm{m}^{2}\right)$, mean $(\mathrm{SD})$ & $41.8(9.7)$ & $38.1(9.3)$ & $40.9(9.8)$ & 0.164 \\
\hline LVH, $n(\%)$ & $9(29)$ & $23(43.4)$ & $39(73.5)^{9 \S}$ & $<0.001$ \\
\hline iLVMI, $n(\%)$ & $11(35)$ & $37(70)^{\dagger}$ & $44(83)^{95}$ & $<0.001$ \\
\hline
\end{tabular}

IVST: interventricular septal thickness; LVPWT: left ventricular posterior wall thickness; LVEDD: left ventricular end-diastolic diameter; LVESD: left ventricular end-systolic diameter; LVM: left ventricular mass; LVMI: left ventricular mass index; LVEF: left ventricular ejection fraction; LVH: left ventricular hypertrophy, defined as LVMI $\geq 134 \mathrm{~g} \mathrm{~m}^{-2}$ in men and $110 \mathrm{~g} \mathrm{~m}^{-2}$ in women; iLVMI: inappropriate LVMI, defined as positive if measured LVMI to predicted LVMI ratio was greater than $135 \%$.

${ }^{\dagger}$ Group 2 versus group 1, $P<0.05$.

${ }^{9}$ Group 3 versus group 1, $P<0.05$.

${ }^{\S}$ Group 3 versus group 2, $P<0.05$.

were performed with SPSS version 16.0 (SPSS, Chicago, IL, USA).

\section{Results}

Thirty-one patients with essential hypertension were enrolled as Group 1. A total of 106 patients with PA were further divided into two groups by the level of aldosterone in the 24-hour urine sample (Group 2, PA patients with 24-hour urinary aldosterone level below median; Group 3, PA patients with 24-hour urinary aldosterone level above median). The baseline clinical characteristics are shown in Table 1. The median level of urinary aldosterone in patients with PA was $9.25 \mu \mathrm{g} /$ day. Patients with PA had higher serum aldosterone concentration, lower plasma renin activity, lower serum potassium level, and higher body mass index. There was a trend for higher diastolic blood pressure in patients with PA compared with those in the essential hypertension group. Concerning medication usage, a higher percentage of patients with PA received ACEI or ARB, alpha blocker, beta-blocker, and spironolactone, compared with the essential hypertension group. There were 23 patients (51\%) and 25 patients $(50 \%)$ receiving aldosterone treatment in group 2 and group 3. There were 30 patients (58\%) versus of 46 patients $(87 \%)$ with aldosterone-producing adenoma in group 2 and group 3 , respectively $(P<0.001)$.

Patients with PA had thicker LV wall thickness, larger LV mass, and a lower LV ejection fraction (Table 2). The E and A wave in these 3 groups were comparable. The $\mathrm{E}^{\prime}$ wave by tissue Doppler from the septum was also lower in patients with PA. The number of patients with LVH and inappropriate LVMI was higher in Groups 2 and 3 compared with Group 1.
In the univariate analysis, LVMI was significantly associated with 24-hour urinary aldosterone level, body mass index, body weight, log-transformed PAC, log-transformed PRA, log-transformed PAC-to-PRA ratio (ARR), systolic and diastolic blood pressure, gender, serum potassium level, the use of alpha-blocker, and the presence of adenoma (Table 3). The correlations of 24-hour urinary aldosterone level, PAC, or ARR with LV structure parameters are shown in Supplementary Table 1 (available online at http://dx.doi.org/10.1155/2013/294594) (group 1, 2, and 3). Compared with PAC, PRA, or ARR, the 24-hour urinary aldosterone level has a better correlation with LV structure parameters. The correlations of 24-hour urinary aldosterone level with LVM, LVMI, measured-to-predicted LVMI ratio, excess LVMI, and ventricular wall thickness were significant in all of the patient groups.

In multivariate analysis, 24-hour urinary aldosterone level, serum potassium level, diastolic blood pressure, and gender were independent factors to predict LVMI in the groups 1, 2, and 3 (Supplementary Table 2). The 24-hour urinary aldosterone level was also a significant predictor for the excess LVMI in whole study population (Supplementary Table 2).

In patients with $\mathrm{PA}$, the univariate analysis revealed that log-transformed urine aldosterone level $(P=0.005)$, serum potassium level $(P=0.006)$, diastolic blood pressure, the presence of adenoma, and alpha blocker were significantly associated with LVMI (Table 4). In the multivariate analysis of LVMI in patients with PA, only log-transformed urine aldosterone level $(P=0.025)$ and serum potassium level $(P=0.037)$ were independent factors for LVMI (Table 5). In the multivariate analysis using excess LVMI as the dependent 
TABLE 3: Factors associated with LVMI in groups 1, 2, and 3 patients.

\begin{tabular}{lcc}
\hline Factor & Pearson's correlation & \multicolumn{1}{c}{$P$} \\
\hline Urine aldosterone & 0.359 & 0.001 \\
Urine aldosterone to plasma renin activity ratio & 0.287 & 0.001 \\
Plasma aldosterone to renin ratio & 0.223 & 0.009 \\
Plasma aldosterone concentration & 0.143 & 0.096 \\
Plasma renin activity & -0.197 & 0.022 \\
Body mass index & 0.026 & 0.016 \\
Body weight & 0.202 & 0.018 \\
Systolic blood pressure & 0.233 & 0.01 \\
Diastolic blood pressure & 0.295 & 0.001 \\
Age & -0.048 & 0.579 \\
Sex & 0.196 & 0.022 \\
Adenoma & 0.264 & 0.007 \\
Serum potassium & -0.354 & 0.001 \\
Alpha blocker & 0.208 & 0.02 \\
\hline
\end{tabular}

TABLE 4: The correlation of urine aldosterone level and LV wall thickness, dimension, and LA size in patients with PA (groups 2 and 3 ).

\begin{tabular}{lccccccccc}
\hline & LVMI & Excess LVMI & LV mass & IVS & PW & LVEDD & LVESD & RWT & LA \\
\hline Urine aldosterone & $0.281^{*}$ & $0.265^{*}$ & $0.286^{*}$ & $0.300^{*}$ & $0.298^{*}$ & 0.149 & 0.037 & 0.150 & 0.170 \\
PAC & 0.020 & 0.057 & 0.019 & 0.064 & 0.094 & -0.069 & -0.061 & -0.031 & 0.029 \\
PRA & -0.046 & -0.077 & 0.012 & -0.043 & -0.036 & 0.099 & 0.078 & -0.179 & -0.041 \\
ARR & 0.050 & 0.090 & -0.005 & 0.061 & 0.064 & -0.115 & -0.093 & 0.157 & 0.048 \\
\hline
\end{tabular}

IVS: interventricular septum; PW: posterior wall; LVEDD: left ventricular end-diastolic diameter; LVESD: left ventricular end-systolic diameter; RWT: relative wall thickness $=2 \times$ PW/LVEDD; LA: left atrium.; PAC: plasma aldosterone concentration; PRA: plasma renin activity; ARR: plasma PAC-to-PRA ratio. ${ }^{*} P<0.05$.

${ }^{\dagger}$ Excess LVMI = measured LVMI - predicted LVMI.

TABLE 5: Predictors for LVMI or inappropriate LVMI in patients with PA (groups 2 and 3).

\begin{tabular}{lcr}
\hline Independent variables & $B(95 \%$ CI $)$ & $P$ \\
\hline (A) LVMI as the dependent factor & & \\
Urine aldosterone & $29.9(8.6-51.3)$ & 0.006 \\
Serum potassium & $-13.4(-23--3.7)$ & 0.007 \\
Diastolic BP & $0.629(0.147-1.112)$ & 0.011 \\
Sex & $13.7(1.15-26.33)$ & 0.033 \\
(B) Excess LVMI as the dependent factor & & 0.003 \\
Urine aldosterone & $29.9(10.6-49.2)$ & 0.003 \\
Serum potassium & $-13.6(-22-4.8)$ & \\
\hline
\end{tabular}

${ }^{\text {Excess LVMI }}=$ measured LVMI - predicted LVMI.

variable, only log-transformed urine aldosterone level was an independent factor in groups 2 and 3 (Table 5).

The performance to predict the presence of $\mathrm{LVH}$ and inappropriate LVMI is shown in Figures 1 and 2. Comparing the accuracy to predict the presence of $\mathrm{LVH}$, the receiver operating characteristic (ROC) area under curve (AUC) of urine aldosterone was larger than that of PAC, especially in the PA patients (in groups 2 and 3: 24-hour urinary aldosterone $\mathrm{AUC}=0.701$ (95\% CI 0.601-0.801) versus $\mathrm{PAC}$ $\mathrm{AUC}=0.568$ (95\% 24 CI 0.456-0.68), $P=0.05$ ). Comparing the accuracy to predict the presence of inappropriate LVMI (measured to predicted LVMI >135\%), the ROC AUC of 24hour urinary aldosterone was also larger than that of PAC or
ARR (in groups 2 and 3: 24-hour urinary aldosterone AUC = 0.61 (95\% CI 0.48-0.734) versus PAC AUC $=0.43$ (95\% CI $0.31-0.551), P=0.028)$. The ROC AUC of 24-hour urinary aldosterone is not significantly higher than that of PAC or ARR in patients with essential hypertension (group 1).

\section{Discussion}

The major findings of our study were (1) the 24-hour urinary aldosterone level was significantly correlated with LV structure parameters in patients with PA; (2) the 24-hour urinary aldosterone level was an independent factor for the LVMI and excess LVMI (not explained by gender, body size, and 


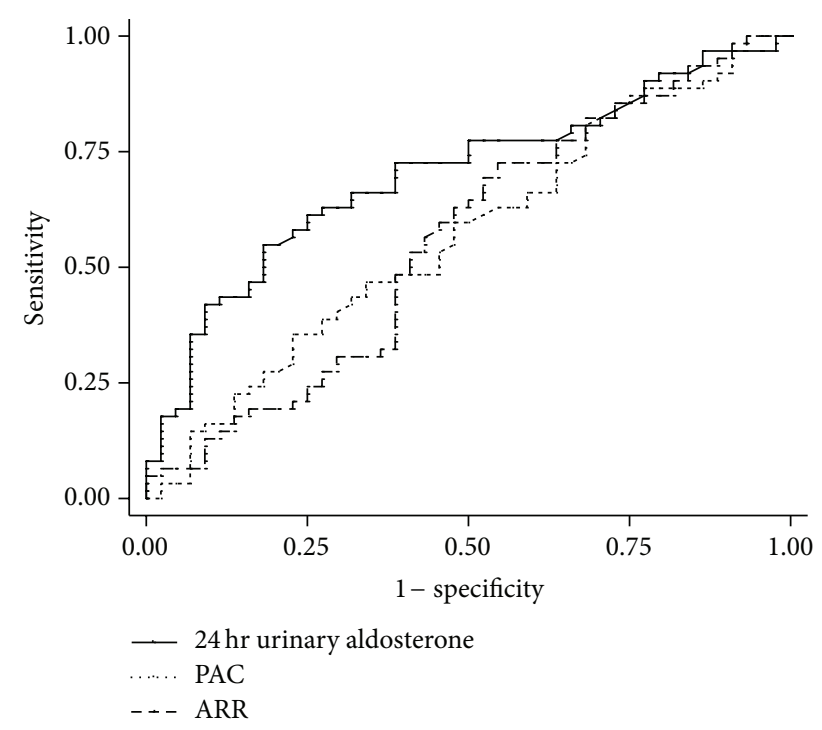

FIGURE 1: ROC curve: to predict the presence of LVH in patients with primary aldosteronism: the ROC AUC of log-transformed $24 \mathrm{hr}$ urinary aldosterone is larger than that of PAC or ARR. (ROC AUC for log-transformed urine aldosterone: 0.701 (95\% CI 0.6010.801), for PAC: $0.568^{\dagger}$ (95\% CI 0.456-0.68), for plasma aldosterone to renin activity ratio: $0.566^{9}(0.445-0.675),{ }^{\dagger} P<0.01{ }^{9} P<0.05$, compared with log-transformed urine aldosterone).

blood pressure) in patients with PA; and (3) the accuracy of 24-hour urinary aldosterone to the presence of inappropriate LVMI was better than that of baseline PAC or ARR in patients with PA.

Although aldosterone has long been connected to excessive LVH in patients with PA, the correlation of baseline plasma aldosterone level and LVMI is not strong in the literature $[4,18]$. In our study, baseline PAC was not significantly related to LVMI and only marginally correlated to the excess LVMI. In contrast, urine aldosterone level was significantly correlated to both LVMI and excess LVMI. In the multivariate analysis, urine aldosterone was repeatedly shown to be an independent factor for LVMI and excess LVMI. In short, urine aldosterone level had higher accuracy to diagnose LVH.

Since plasma aldosterone level is influenced by many factors, such as sodium intake, medication [19], or circadian rhythm [20], a single time point plasma level might not have a good correlation with the chronic exposure of myocardium to aldosterone over a long period. The 24 -hour urinary aldosterone level may reflect the exposure of aldosterone more accurately than baseline PAC. Our observation is in line with previous research on patients with essential hypertension; while the 24-hour urinary aldosterone level was significantly correlated with LV mass, the PAC was not significantly correlated with LV structure in patients with essential hypertension [10]. Although 24-hour urinary aldosterone has been found to be correlated with inappropriate LVMI in the study of Muiesan et al., the correlations between 24-hour urinary aldosterone with LVMI and other LV structural parameters were not available in this study [4]. The relative performance

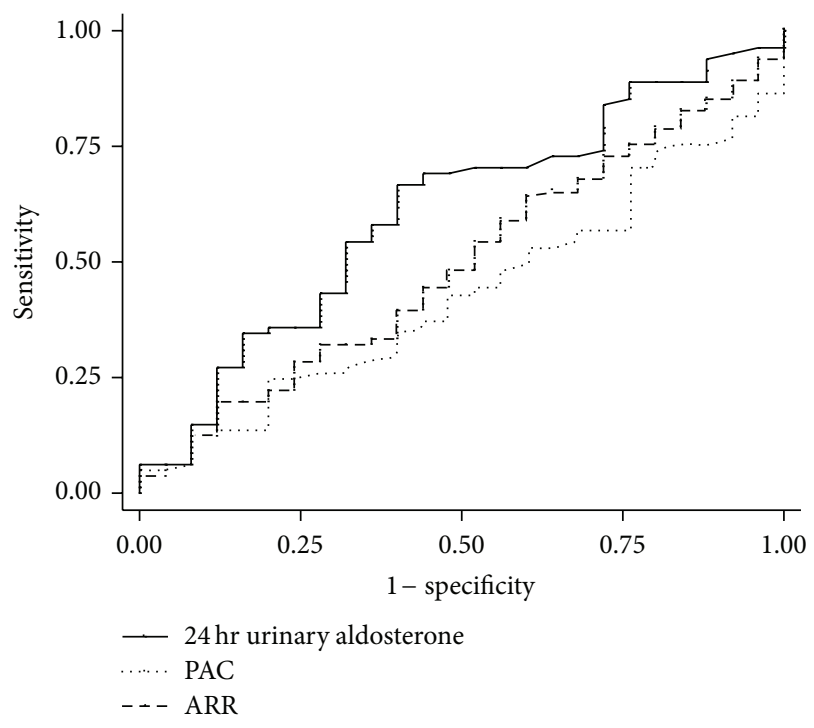

FIGURE 2: ROC curve: to predict the presence of inappropriate LVMI in patients with primary aldosteronism: the ROC AUC of logtransformed $24 \mathrm{hr}$ urinary aldosterone is larger than that of PAC or ARR. (ROC AUC for log-transformed $24 \mathrm{hr}$ urine aldosterone: 0.61 (95\% CI 0.48-0.734); for PAC: 0.43 (95\% CI 0.31-0.551); for plasma aldosterone to renin activity ratio: 0.493 (95\% CI 0.36-0.61), ${ }^{\dagger} P<0.01$ compared with log-transformed urine aldosterone) The presence of inappropriate LVMI is defined as positive if measured to predicted LVMI ratio $>135 \%$.

between plasma aldosterone and 24-hour urinary aldosterone was shown in our study. Our data clearly revealed that 24-hour urinary aldosterone level had a better performance to identify LV structural change in patients with PA. There are some possible explanations for the differences in the results. First, the prevalence of LVH may be slightly higher in our population ( $\mathrm{LVH} n=71,52 \%)$. As the duration from disease onset to the performance of the previous study was unknown, it is possible that the correlation may be stronger in a population with a longer established hypertrophy. Second, the number of patients with adenoma was also higher in our population (adenoma, $n=76,71 \%$ of groups 2 and 3 ). We cannot rule out the correlations between patients with adenoma and patients with hyperplasia are different.

Patients with PA are associated with a high cardiovascular complication rate disproportional to their blood pressures [21]. The LV mass of patients with PA has also been shown to be out of proportion to the extent that can be induced by hemodynamic load and body size $[3,4]$. These two pieces of evidence affirm the importance of looking at the inappropriateness of $\mathrm{LV}$ mass in patients with PA and the translation of these changes into excessive cardiovascular events. In patients with essential hypertension, the inappropriateness of LV mass has been shown to predict cardiovascular risk independently of age and blood pressure $[8,22]$. According to our observation, the presence of inappropriate LVMI may occur before the presence of LVH. Whereas data concerning the long-term prognostic value of the presence of inappropriate LVMI in patients with PA is still pending, based on our observation, it 
would be reasonable to monitor 24-hour urinary aldosterone level for future cardiovascular complications.

Aldosterone has been shown to have a direct hypertrophic effect on cardiomyocytes. In neonatal rat ventricular myocytes, aldosterone caused a $27 \%$ increase in protein incorporation and a $29 \%$ increase in cardiomyocyte surface area. This was also associated with increased mRNA levels of $\alpha$-and $\beta$-myosin heavy chain [23]. Furthermore, aldosterone increases interstitial fibrosis in myocardium. In vitro data revealed that aldosterone increases the collagen synthesis in cardiac fibroblast [24]. In our previous report using integrated backscatter on echocardiography, the increased amount of collagen content in patients with PA could be reversed after adrenalectomy [13].

There are several limitations in this study. First, the small patient number in this study prevents us from drawing definite conclusions. A further large-scale study is needed to confirm our results. Second, this is a cross-sectional study without follow-up data. Whether urinary aldosterone can predict long-term cardiovascular events better than other parameters is still unknown. Third, the data after operation are not available in this study. Further study to investigate the urinary aldosterone level and the regression in LVH and inappropriate LVMI after surgery is warranted. Fourth, the aldosterone is secreted mainly as tetrahydroaldosterone and $10 \%$ as aldosterone-18-glucuronide and less than $5 \%$ aldosterone is secreted in the free aldosterone. Other aldosterone metabolites are unknown or seldom routinely measured. We did not check the tetrahydroaldosterone level in our study, mainly because of the cost. However, our results based on the urinary aldosterone level provide a simple and feasible way to identify LVH and inappropriate LVMI in clinical practice. Fifth, the urinary sodium level was not measured in our study. The relationship between urinary sodium, $24 \mathrm{hr}$ urinary aldosterone level, and LVH could not be determined. The effect of the antihypertensive drugs effect also could not be fully excluded. Finally, although the 24-hour urinary aldosterone level is the only factor that significantly correlated with left ventricular structure parameters in patients with essential hypertension (Supplementary Table 1), the performance to identify the presence of LVH or inappropriate LVMI was not significantly higher than that for PAC or ARR in this patient group. This statistical nonsignificance may be due to the small number of patients in this group.

\section{Conclusion}

In conclusion, our results show that 24-hour urinary aldosterone is significantly correlated with LV structural parameters including LV wall thickness and LV mass. The inappropriate LV mass, exceeding the amount required to compensate for the loading condition, is also correlated with 24hour urinary aldosterone. The 24-hour urinary aldosterone is a better indicator for the presence of inappropriate LVMI in patients with PA compared with PAC or ARR.

\section{Conflict of Interests}

The authors declare that they have no conflict of interests.

\section{Acknowledgments}

This study was supported by the Ta-Tung Kidney Foundation, National Taiwan University Hospital (NTUH. 99M1427), the Taiwan National Science Council (Grant NSC 97-2314-B-002-155-MY2, NSC 100-2314-B-002-139), and the Taiwan National Science Council support for the Center for Dynamical Biomarkers and Translational Medicine, National Central University, Taiwan (NSC 100-2911-I-008-001). The funders had no role in study design, data collection and analysis, decision to publish, or preparation of the paper. The authors would like to thank the staff of the Second Core Lab of Department of Medical Research in National Taiwan University Hospital for technical assistance. Taiwan Primary Aldosteronism Investigation (TAIPAI) Study Group: Vin-Cent Wu, M.D., Ph.D., Yen-Hung Lin, M.D., Ph.D., YiLuwn Ho, M.D., Ph.D., Hung-Wei Chang, M.D., Ph.D., LianYu Lin, M.D., Ph.D., Fu-Chang Hu, M.S., Sc.D., Kao-Lang Liu, M.D., Shuo-Meng Wang, M.D., Kuo-How Huang, M.D., Ph.D., Yung-Ming Chen, M.D., Chin-Chi Kuo, M.D., ShihChieh Chueh, M.D., Ph.D., Ching-Chu Lu, M.D., Fang-Chi Chang, M.D., Shih-Cheng Liao, M.D., Ruoh-Fang Yen, M.D., Ph.D., Wei-Chou Lin, M.D., Ph.D., Bor-Sen Hsieh, M.D., Ph.D. and Kwan-Dun Wu, M.D., Ph.D.

\section{References}

[1] G. P. Rossi, T. M. Seccia, and A. C. Pessina, "Primary aldosteronism-part I: prevalence, screening, and selection of cases for adrenal vein sampling," Journal of Nephrology, vol. 21, no. 4, pp. 447-454, 2008.

[2] G. P. Rossi, "Prevalence and diagnosis of primary aldosteronism," Current Hypertension Reports, vol. 12, no. 5, pp. 342-348, 2010.

[3] G. P. Rossi, A. Sacchetto, P. Visentin et al., "Changes in left ventricular anatomy and function in hypertension and primary aldosteronism," Hypertension, vol. 27, no. 5, pp. 1039-1045, 1996.

[4] M. L. Muiesan, M. Salvetti, A. Paini et al., "Inappropriate left ventricular mass in patients with primary aldosteronism," Hypertension, vol. 52, no. 3, pp. 529-534, 2008.

[5] P. J. Fuller and M. J. Young, "Mechanisms of mineralocorticoid action," Hypertension, vol. 46, no. 6, pp. 1227-1235, 2005.

[6] P. Lijnen and V. Petrov, "Induction of cardiac fibrosis by aldosterone," Journal of Molecular and Cellular Cardiology, vol. 32, no. 6, pp. 865-879, 2000.

[7] G. de Simone, R. B. Devereux, T. R. Kimball et al., "Interaction between body size and cardiac workload: influence on left ventricular mass during body growth and adulthood," Hypertension, vol. 31, no. 5, pp. 1077-1082, 1998.

[8] V. Palmieri, K. Wachtell, E. Gerdts et al., "Left ventricular function and hemodynamic features of inappropriate left ventricular hypertrophy in patients with systemic hypertension: The LIFE Study," American Heart Journal, vol. 141, no. 5, pp. 784-791, 2001.

[9] Y. H. Lin, S. M. Wang, V. C. Wu et al., "The association of serum potassium level with left ventricular mass in patients with primary aldosteronism," European Journal of Clinical Investigation, vol. 41, no. 7, pp. 743-750, 2011.

[10] Y. Jin, T. Kuznetsova, M. Maillard et al., "Independent relations of left ventricular structure with the 24-hour urinary excretion of sodium and aldosterone," Hypertension, vol. 54, no. 3, pp. 489-495, 2009. 
[11] C. Delles, B. M. W. Schmidt, H. J. Muller, S. Oehmer, A. U. Klingbeil, and R. E. Schmieder, "Functional relevance of aldosterone for the determination of left ventricular mass," The American Journal of Cardiology, vol. 91, pp. 297-230, 2003.

[12] V.-C. Wu, S.-C. Chueh, H.-W. Chang et al., "Association of Kidney Function with residual hypertension after treatment of aldosterone-producing adenoma," American Journal of Kidney Diseases, vol. 54, no. 4, pp. 665-673, 2009.

[13] Y.-H. Lin, H.-H. Lee, K.-L. Liu et al., "Reversal of myocardial fibrosis in patients with unilateral hyperaldosteronism receiving adrenalectomy," Surgery, vol. 150, no. 3, pp. 526-533, 2011.

[14] V. C. Wu, S. Y. Yang, J. W. Lin et al., "Kidney impairment in primary aldosteronism," Clinica Chimica Acta, vol. 412, pp. 1319-1325, 2011.

[15] R. M. Lang, M. Bierig, R. B. Devereux et al., "Recommendations for chamber quantification: a report from the American Society of Echocardiography's Guidelines and Standards Committee and the Chamber Quantification Writing Group, developed in conjunction with the European Association of Echocardiography, a branch of the European Society of Cardiology," Journal of the American Society of Echocardiography, vol. 18, no. 12, pp. 1440-1463, 2005.

[16] R. B. Devereux and N. Reichek, "Echocardiographic determination of left ventricular mass in man. Anatomic validation of the method," Circulation, vol. 55, no. 4, pp. 613-618, 1977.

[17] R. B. Devereux, "Detection of left ventricular hypertrophy by M-mode echocardiography. Anatomic validation, standardization, and comparison to other methods," Hypertension, vol. 9, no. 2, pp. II19-II26, 1987.

[18] E. Pimenta, R. D. Gordon, A. H. Ahmed et al., "Cardiac dimensions are largely determined by dietary salt in patients with primary aldosteronism: results of a case-control study," Journal of Clinical Endocrinology and Metabolism, vol. 96, no. 9, pp. 2813-2820, 2011.

[19] P. Mulatero, F. Rabbia, A. Milan et al., "Drug effects on aldosterone/plasma renin activity ratio in primary aldosteronism," Hypertension, vol. 40, no. 6, pp. 897-902, 2002.

[20] D. C. Kem, M. H. Weinberger, and C. Gomez Sanchez, "Circadian rhythm of plasma aldosterone concentration in patients with primary aldosteronism," The Journal of Clinical Investigation, vol. 52, no. 9, pp. 2272-2277, 1973.

[21] P. Milliez, X. Girerd, P.-F. Plouin, J. Blacher, M. E. Safar, and J.-J. Mourad, "Evidence for an increased rate of cardiovascular events in patients with primary aldosteronism," Journal of the American College of Cardiology, vol. 45, no. 8, pp. 1243-1248, 2005.

[22] G. de Simone, V. Palmieri, M. J. Koren, G. A. Mensah, M. J. Roman, and R. B. Devereux, "Prognostic implications of the compensatory nature of left ventricular mass in arterial hypertension," Journal of Hypertension, vol. 19, no. 1, pp. 119-125, 2001.

[23] M. P. Okoshi, X. Yan, K. Okoshi et al., "Aldosterone directly stimulates cardiac myocyte hypertrophy," Journal of Cardiac Failure, vol. 10, no. 6, pp. 511-518, 2004.

[24] K. Rombouts, A. Wielant, K. Hellemans, D. Schuppan, and A. Geerts, "Influence of aldosterone on collagen synthesis and proliferation of rat cardiac fibroblasts," British Journal of Pharmacology, vol. 134, no. 1, pp. 224-232, 2001. 


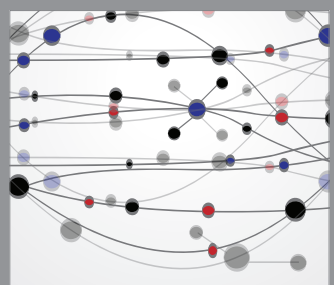

The Scientific World Journal
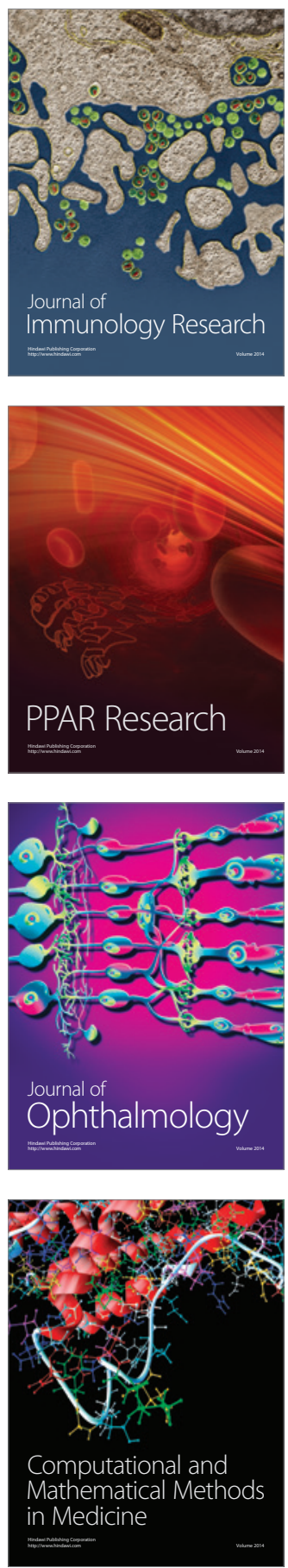

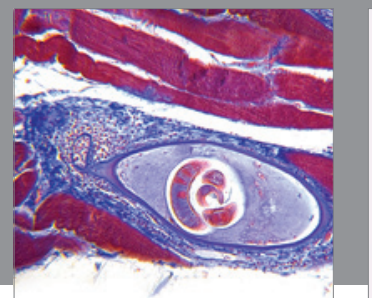

Gastroenterology

Research and Practice
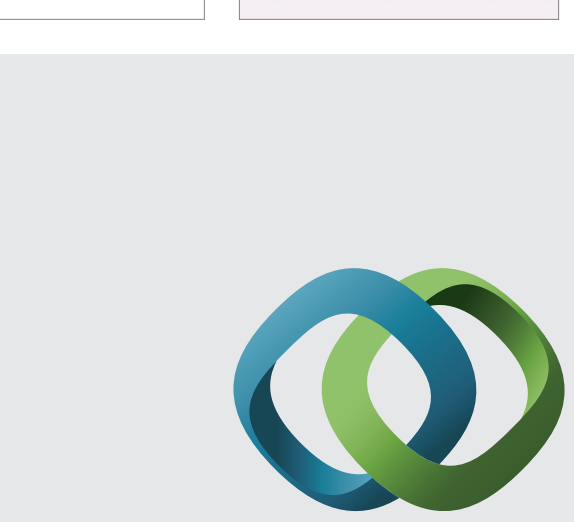

\section{Hindawi}

Submit your manuscripts at

http://www.hindawi.com
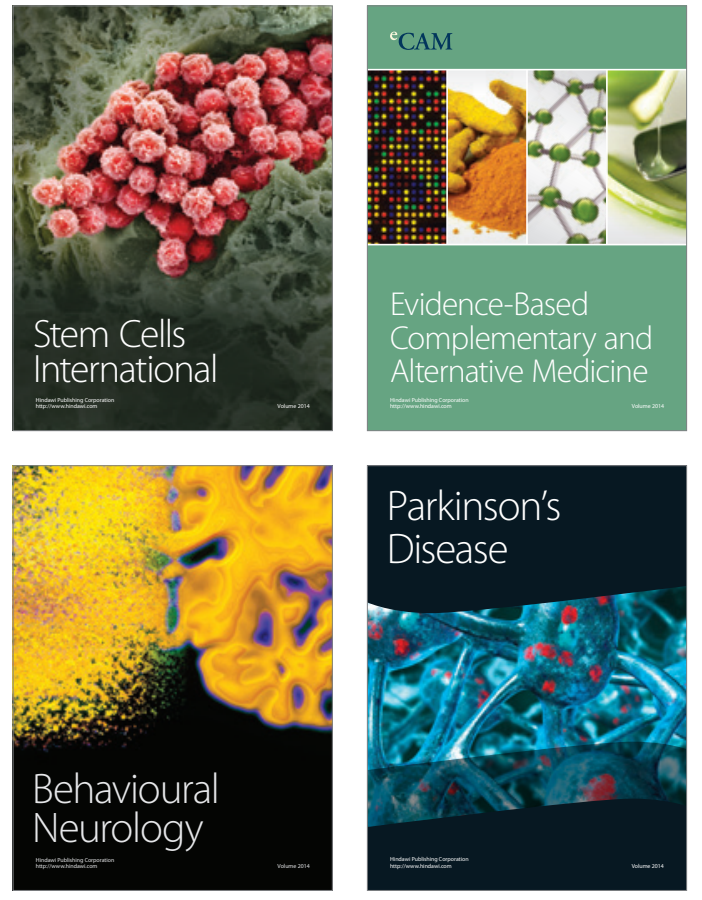
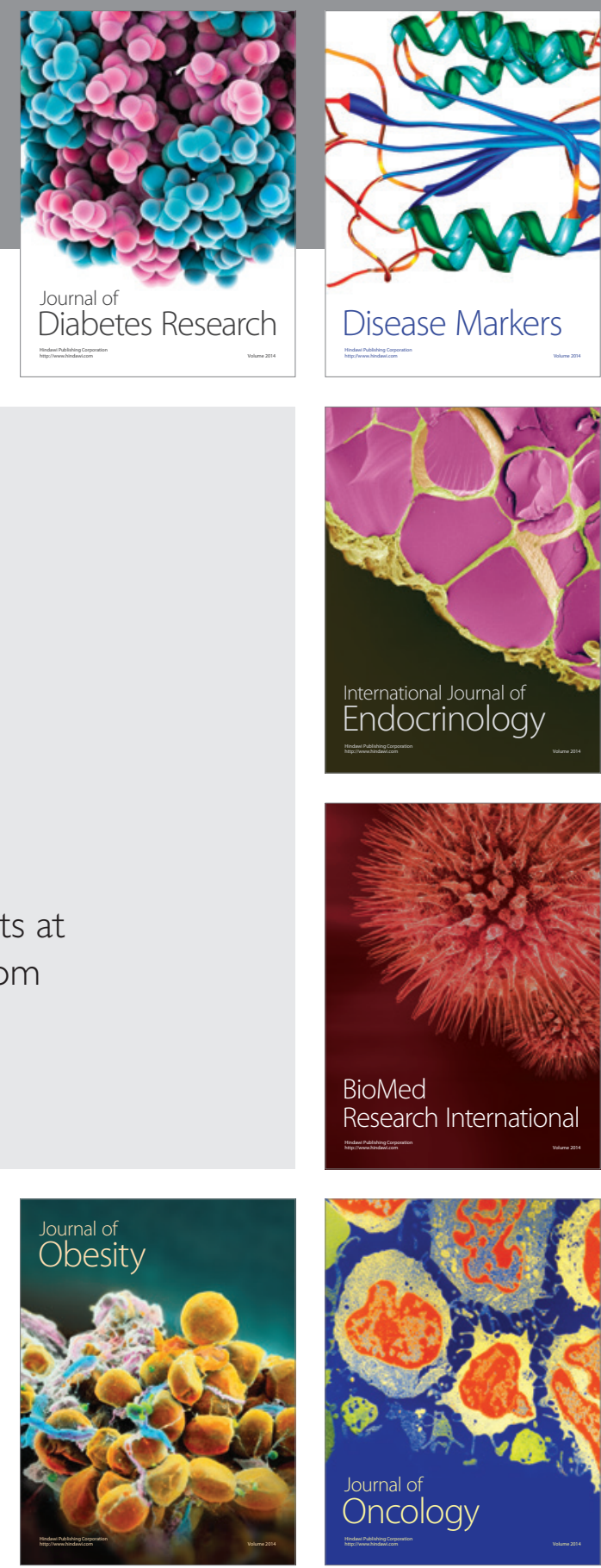

Disease Markers
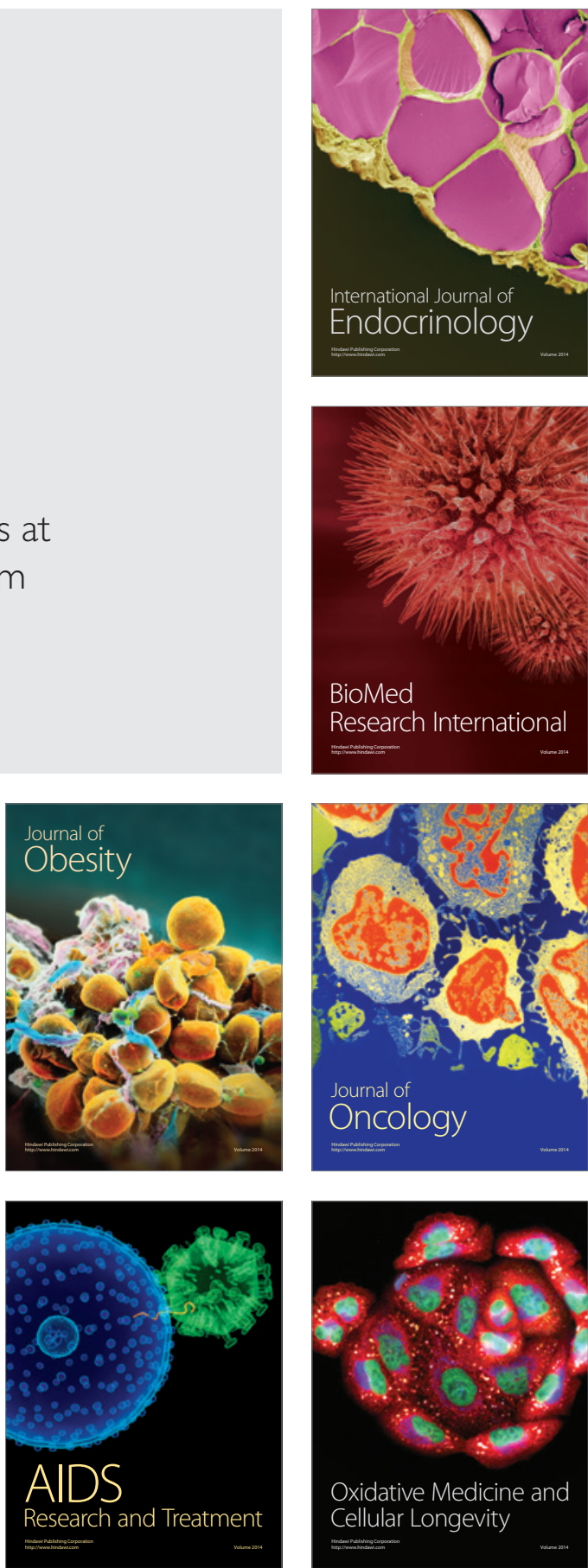